\title{
Profiles of children's physical activity and sedentary behaviour between age 6 and 9: a latent profile and transition analysis
}

\author{
Russell Jago ${ }^{1 *}$ (D), Ruth Salway ${ }^{1}$, Deborah A. Lawlor ${ }^{2,3}$, Lydia Emm-Collison ${ }^{1}$, Jon Heron ${ }^{3}$, Janice L. Thompson ${ }^{4}$
} and Simon J. Sebire ${ }^{1}$

\begin{abstract}
Background: Physical activity is associated with improved physical and mental health among children. However, physical activity declines and sedentary time increases with age, and large proportions of older children do not meet the recommended hour per day of moderate-to-vigorous-intensity physical activity (MVPA). The aim of this paper is to identify profiles of children based on the complex relationship between physical activity and sedentary time at ages 6 and 9 and explore how those profiles are associated with other covariates and how they change over time.

Methods: Valid accelerometer data were collected for 1132 children aged 6 and 1121 at age 9, with 565 children with data at both ages. We calculated the proportions of total wear time spent in sedentary, light and MVPA activity on both weekdays and weekends. Latent profile (class) analysis was applied separately to the two age groups to identify activity profiles. We then used latent transition analysis to explore transitions between profiles at the two time points.

Results: We identified five profiles of activity at age 6 and six profiles at age 9. Although profiles were not directly equivalent, five classes captured similar patterns at both ages and ranged from very active to inactive. At both ages, active profiles, where the majority achieved the recommended MVPA guidelines, were more likely to be active at weekends than on weekdays. There was substantial movement between classes, with strongest patterns of movement to classes with no change or a decrease in MVPA. Transition between classes was associated with sex, BMI z-score, screen-viewing and participation in out-of-school activities.

Conclusions: This paper is the first to apply latent profile analysis to the physical activity of UK children as they move through primary school. Profiles were identified at ages 6 and 9, reflecting different weekday and weekend patterns of physical activity and sedentary time. There was substantial movement between profiles between ages 6 and 9, mostly to no change or less active profiles. Weekend differences suggest that greater focus on how weekend activity contributes to an average of 60 min per day of MVPA across the week may be warranted.
\end{abstract}

Keywords: Physical activity, Profile, Transition, Cohort, Children, Weekend

\section{Background}

Physical activity is associated with improved physical and mental wellbeing among children and young people [1]. There is also some evidence that sedentary behaviour is associated with adverse health outcomes among children and young people but there is currently some debate as to whether these associations are independent of physical

\footnotetext{
* Correspondence: russ.jago@bristol.ac.uk

${ }^{1}$ Centre for Exercise, Nutrition \& Health Sciences, School for Policy Studies,

University of Bristol, 8 Priory Road, Bristol BS8 1TZ, UK

Full list of author information is available at the end of the article
}

activity $[2,3]$. The amount of physical activity in which children engage declines as they move through childhood and into adulthood, with large proportions of older children and adolescents not engaging in the recommended hour per day of moderate-to-vigorous-intensity physical activity (MVPA) [4, 5]. Conversely, large-scale international studies have shown that sedentary behaviour increases as children age [4]. Increasing physical activity [6] and reducing sedentary time [7] are both issues of global importance but attempts to increase children's physical activity and reduce sedentary time have had limited

(C) The Author(s). 2018 Open Access This article is distributed under the terms of the Creative Commons Attribution 4.0 International License (http://creativecommons.org/licenses/by/4.0/), which permits unrestricted use, distribution, and 
impact, suggesting that new ways of helping children to be more active and less sedentary are required [8].

The relationship between physical activity and sedentary behaviour is complex [9]. While sedentary behaviour is often defined (academically) as not simply a state of physical inactivity, but a separate and distinct behaviour, parents often view reducing sedentary behaviour as key to increasing their child's physical activity and vice versa [10]. Several international bodies have suggested that movement behaviours should be considered that integrate physical activity and sedentary time across the day rather than focusing on just one behaviour $[11,12]$. Furthermore, several studies have suggested that re-allocating sedentary time to light intensity physical activity would elicit reductions in the risk factor profile of children and adults $[13,14]$. Thus, there is a need to consider overall movement behaviour profiles of children to identify if there are patterns of behaviour that: a) may be associated with reduced health risk; b) show a less steep decline in overall physical activity from childhood to adolescence; and c) offer insights into how to help children reach and maintain sufficient levels of activity to promote health.

Cluster analysis [15] and latent class analysis [16] are methods used to identify groups of people who share similar characteristics. These methods are especially useful when characteristics combine in complex ways and therefore applying these to children's physical activity and sedentary behaviours could help to increase our understanding of these behaviours. Evidence-based cluster analysis may define groups based on health-related cut-points, whereas more data-driven methods use the data themselves to determine appropriate clusters. Unlike cluster analysis, latent class analysis uses an underlying probabilistic model which means that the uncertainty in class membership can be estimated and included in standard statistical techniques [16]. Traditionally, latent class analysis (LCA) refers to the situation where these variables are categorical, and latent profile analysis (LPA) when they are continuous although, in practice, there is not such a clear distinction and it is possible to combine categorical and continuous variables [17]. Latent class analysis has been used in other public health applications such as substance use, smoking, exercise and dietary behaviour [18-21], but has been less common in studies of physical activity, especially among children. A recent review [22] found that the majority of studies in the physical activity context used cluster analysis. These cluster analyses were mainly cross-sectional studies involving older children ( $>9$ years) or adolescents, and consistently found girls identified within clusters characterised by low physical activity. Among studies applying LCA, one cross-sectional study used objective accelerometer data to measure physical activity in children under 11 [23], and MVPA and sedentary time were analysed separately thus not including interactions between different types of activity. To date, longitudinal studies have used the same fixed classes at different time points, and thus not considered how classes might have changed over time. A large cohort analysis [24] using self-report physical activity data in adolescents aged 11-21 reported overall changes in the distribution of classes but did not look at movement between classes. A much smaller study [25] used accelerometer data in children 5-6 and 10-12 and reported change between profiles, but sample sizes were small and did not allow any investigation of factors associated with transitions. There are no studies in younger children using accelerometer-measured MVPA and sedentary time that look at whether the activity profiles change over time or seek to identify factors that are associated with movement between profiles.

The aim of this paper is to identify profiles of children based on the complex relationship between physical activity and sedentary time at ages 6 and 9 and explore how those profiles may change over time and how children move between them. In addition, we aim to explore how these profiles and transitions are associated with factors such as sex, BMI, deprivation and activities.

\section{Methods}

Data are from the B-PROACT1V study, a longitudinal study that aimed to examine the physical activity and sedentary behaviours of primary school children aged 511 years, and their parents [5, 26, 27]. The study received ethical approval from the School of Policy Studies Ethics Committee at the University of Bristol, UK, and written parental consent was received for all participants [28]. In Phase 1, all children in Year 1 of primary school (aged 5-6 years) from 57 schools in and around Bristol were invited to participate, with data collection taking place between January 2012 and July 2013. In Phase 2, when the children were in Year 4 (aged 8-9 years), all schools from Phase 1 were invited to participate, with 47 schools agreeing. All children were eligible to participate regardless of whether they had participated in Phase 1, and data collection took place between March 2015 and July 2016. Data were collected for 1299 children in Year 1 and 1223 children in Year 4, with 685 children included in both phases.

\section{Accelerometer data}

Children wore a waist-worn ActiGraph wGT3X-BT accelerometer for 5 days, including two weekend days. Accelerometer data were processed using Kinesoft (v3.3.75; Kinesoft, Saskatchewan, Canada) and analysis was restricted to those children who provided at least 2 days of valid weekday data and one valid weekend day to 
provide a compromise between a typical day and maximising the sample size. A valid day was defined as at least $500 \mathrm{~min}$ of data, after excluding intervals of $\geq 60 \mathrm{~min}$ of zero counts allowing up to 2 min of interruptions [4]. Valid data were available for 1132 children at age 6, 1121 at age 9 and 565 at both ages. Data were recorded at $10 \mathrm{~s}$ intervals and characterised as sedentary, light or MVPA using Evenson population-specific cut points for children [29]. The average number of MVPA and sedentary minutes per day were derived for each child, and average minutes for weekday and weekend were calculated. Wear times differed between children, depending on the time of year of data collection and between ages 6 and 9. To avoid wear-time related bias in the latent classes, we used the proportion of total wear time spent in sedentary, light and MVPA activity.

\section{Other measurements}

Child height and weight were measured, and body mass index (BMI) was calculated and converted to an ageand sex-specific standard deviation score based on UK reference curves $[30,31]$. Indices of Multiple Deprivation (IMD) scores, based on the English Indices of Deprivation (http://data.gov.uk/dataset/index-of-mul tiple-deprivation), were assigned to each child based on their reported home postcode. Higher IMD scores indicate a greater level of deprivation.

To understand the contribution of specific domains of physical activity and sedentary behaviour to different physical activity profiles, further details of screen-viewing and activity were obtained. In both years, parents were asked about the number of hours their child typically spent in various screen-viewing activities on weekdays and at weekends (e.g. TV, tablets \& games consoles, coded from $0=$ 'None' to $5=$ ' $4 \mathrm{~h}$ or more') and these were combined to give a total number of average hours spent in screen-viewing on weekdays and weekends. Children completed a short questionnaire, in which they were asked about the frequency (coded from $0=$ 'Never' to $3=$ ' 5 days per week') with which they engaged in different forms of activity outside school hours: sport or exercise club at school, sport or exercise club elsewhere, playing outdoors in their neighbourhood, and playing outdoors at home [32]. These were combined to form three variables: a score 0-6 representing participation in structured activity (clubs), a score 0-6 representing unstructured activity (playing) and a total activity participation score from 0 to 12. In all cases, a higher value indicates a higher frequency of participation in activities outside school. This activity participation variable provides information on the type of activity rather than intensity, and has been shown to be an important predictor of activity with patterns that differ between girls and boys [32].

\section{Statistical analysis}

\section{Latent profile and latent transition analysis}

Latent class analysis is a latent variable model that can be used to identify underlying homogenous subgroups in a population, based on one or more observed variables which may be categorical or continuous; in the latter case the analysis is often called latent profile analysis [17] or finite mixture modelling [33]. Individuals are assumed to belong to one of a set of mutually exclusive latent (unobserved) classes, and in latent profile analysis the observed variables are assumed to be normally-distributed within these classes. As the underlying model is probabilistic, latent profile analysis estimates the parameters of the within-class distributions, and probabilities of class membership for participants. This probabilistic assignment of participants into classes enables uncertainty in class-membership to be appropriately modelled and represents a benefit of the latent approach over methods such as cluster analysis. Latent class analysis allows participants to be included in analyses as long as they have one measure (i.e. they can have partial missing data) through the use of Maximum Likelihood estimation under the assumption that data are missing at random.

Latent transition analysis [16] is a longitudinal extension of latent class/profile analysis requiring the estimation of a latent class model at two or more time-points. Transitions between these time points are modelled via a transition matrix that describes the movement between states through time. With a standard latent class analysis, the focus is typically on the use of explanatory variables to predict class membership (handled via a multinomial regression model). With latent transition analysis, both class membership (time 1 and time 2) and also the transition probabilities may depend on explanatory variables (for instance, a baseline predictor may increase the probability of a participants moving from a high- to low-activity state). All analysis was performed using Mplus v8 [34].

\section{Cross-sectional models}

We fitted cross-sectional latent profile analysis models for age 6 and 9 separately, using as class variables the proportions of time spent in MVPA and sedentary time for weekdays and weekends (light activity is implicitly included as all three proportions must sum to one). We assumed that the weekday and weekend sedentary proportion variances were the same within a class (and likewise for the MVPA variances) but allowed sedentary variances to differ from the MVPA variances, and both to differ across classes. Latent profile models usually assume conditional independence which is theoretically violated here as the proportions are correlated. To account for this, we allowed a residual covariance between weekday sedentary and MVPA proportions and 
the same for weekend proportions. We explored a few alternative specifications to assess the sensitivity of the final models to these assumptions, especially concerning the variance.

There is no single commonly-accepted criterion to determine the number of classes [35], so we used a mixture of statistical criteria, interpretability and parsimony. We initially fit latent profile models for 2-10 classes, and reported the Bayesian Information Criterion [36] (BIC), where a lower value indicated better model fit, and the Lo-Mendell-Rubin (LMR) [37] and bootstrapped likelihood ratio (BLRT) tests [33]. These tests both compare a $\mathrm{k}-1$ versus a $\mathrm{k}$-class model, with a low $p$-value rejecting the $k-1$ class model in favour of the $k$ class model. These criteria have been shown to perform well at identifying an appropriate number of classes [35, 38]. We also reported the relative entropy, an overall measure of classification on a scale of 0 (random) to 1 (perfect classification) [39] and the smallest class size, to identify problematic models with very small class sizes. We considered the interpretability of the final classes in terms of physical activity behaviour and chose a smaller number of classes when all other considerations are equal. Latent variable models were rerun with multiple start values to ensure that the maximum log-likelihood value was replicated.

Once the number of classes was chosen based on the steps outlined above, we applied descriptive labels to each class based on the estimated profile of sedentary/ light/MVPA proportions within each class. Finally, to aid interpretation, we also estimated the expected proportion in each class meeting physical activity guidelines (MVPA $>60 \mathrm{~min}$, based on average wear-time) based on the estimated parameters in each class. Once the classes had been identified we examined whether there were differences between them in terms of sex, standardised BMI z-score, IMD, number of hours of screen viewing and participation in out-of-school activities (for age 9 only) with a Wald test using the $\mathrm{BCH}$ method, which includes the classification error and is robust to assumption violations [40].

\section{Longitudinal model}

A latent transition model [41-43] was used to examine change in class membership for the 565 children who have valid data at both time points. This process combines a cross-sectional estimate of the latent classes at age 6 with a longitudinal description of change over time between ages 6 and 9. Constraining the latent classes to be the same at both time points (measurement invariance) would aid interpretation, so we fit both measurement invariance and non-invariance models to explore whether this assumption is valid. We used a 3-step approach $[42,43]$ to investigate relationships between the latent classes and explanatory variables. This separates the estimation of the latent classes from the larger structural equation model and ensures that the latent classes do not change when including transitions and/or covariates, whilst still accounting for the measurement error in class assignment. Transition probabilities were modelled as a function of each covariate individually.

\section{Missing data}

A total of 1690 children with valid accelerometer data at at least one time point were included. The model for age 6 was based on 1087 weekday accelerometer measurements and 980 weekend measurements, and the model for age 9 was based on 1059 weekday and 942 weekend measurements. Missing covariate information (Additional file 1: Table S1) varied from < 1\% (z-BMI score at age 9) to $18 \%$ (activity participation score at age 9 ), with a total of 964 participants $(80 \%)$ at age 6 and 922 cases $(79 \%)$ at age 9 having complete data, and 449 (66\%) with complete data at both time points.

Although there are missing outcome and covariable data in this study it was not possible to use standard multiple imputation techniques [44] as the latent structure of the classes is unknown and as such each imputation could produce different classes. Instead, we used full information maximum likelihood, which uses available information from all participants and handles missing data within the analysis model, assuming that data is missing at random. This has been shown to produce unbiased parameter estimates and standard errors in structural equation models when data are missing at random [45].

\section{Results}

Participant characteristics of the data at ages 6 and 9 are summarised in Additional file 1: Table S1. Average MVPA on weekdays decreased by 5.3 mins (95\% CI: 3.4 to 7.2 mins) between age 6 and 9 and average weekday sedentary time increased by 73.2 mins (95\% CI: 67.2 to 79.3 mins). There are similar differences in weekend activity with an average decrease of 3.4 mins (95\% CI: 0.1 to $6.6 \mathrm{mins}$ ) and increase of $64.6 \mathrm{mins}$ (95\% CI: 55.3 to 73.9 mins) for MVPA and sedentary time respectively.

\section{Age 6 cross-sectional latent profiles}

Additional file 1: Table S2 reports indicators of model fit for 2-10 classes. The 6-class model had the lowest BIC but identified one very small class (1\%) of very high-sedentary low-MVPA outliers. These outliers appear to be heavily influencing the classes in some models, so we excluded them $(n=4)$ in a sensitivity analysis and found a 5-class model fitted best, with class profiles very similar to the remaining 5 classes from the full data. This suggested that the main influence of the outliers was the formation of the extra class. As this 
class was too small for further analysis and caused identification and interpretation problems in further analyses, we have reported the 5-class model without outliers in the remainder of the paper $(n=1128)$. The full 6 -class model that included the outliers is described in Additional file 1: Table S3 for comparison. We explored several alternative model specifications and found that, while the exact number of classes differed, similar types of profile kept arising, providing support for the 5-class model.

\section{Latent profiles at age 6}

The weekday and weekend profiles for each class are shown in Fig. 1, and the estimated proportions of children in each class are shown in Fig. 3 (left panel; see also Additional file 1: Table S3).

To aid interpretation we have organised the classes approximately in order from most active (highest MVPA) to least active (MVPA). The five classes were identified as follows:

- Highly active (9\%): High and very high levels of MVPA, especially at weekends, combined with low sedentary proportion. Nearly all meet the recommended level of MVPA of 60+ mins/day (92\% on weekdays and $100 \%$ on weekends).

- Active/light (29\%): Higher MVPA than average and average to low sedentary proportion; a large proportion of non-sedentary time is light activity. Most meet the recommended MVPA (77\% on weekdays and $87 \%$ on weekends).

- Active/sed (19\%): Higher MVPA than average (similar to Active/light) but above average sedentary proportion. Most meet the recommended MVPA (77\% and $86 \%)$.

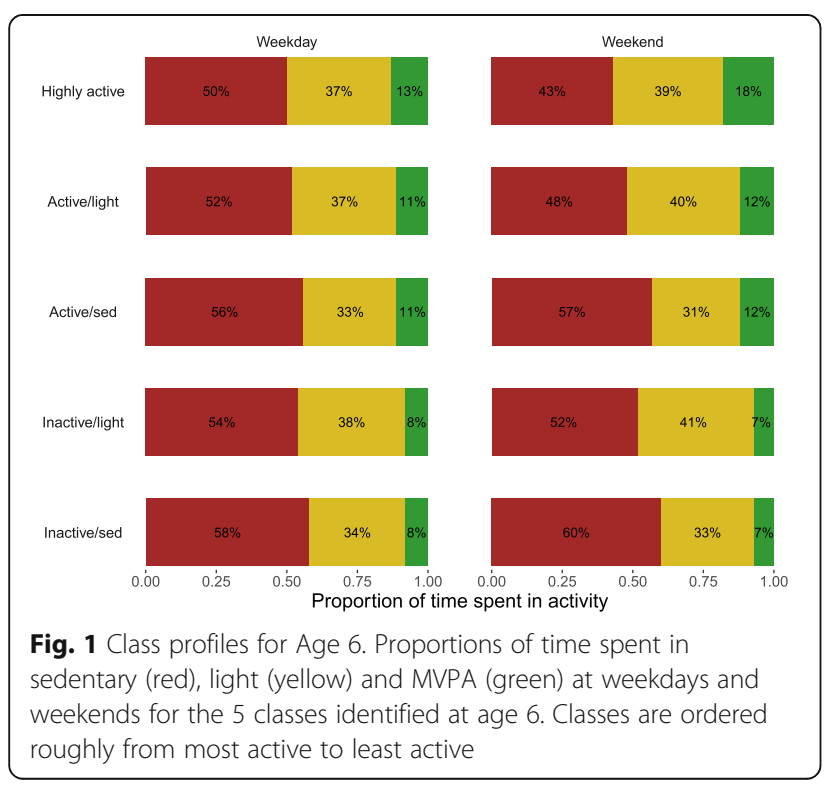

- Inactive/light (15\%): Low MVPA combined with average sedentary proportion; a large proportion of non-sedentary time is light activity. A minority met the recommended MVPA (33\% on weekdays and $19 \%$ on weekends)

- Inactive/sed (28\%): Low MVPA (similar to Inactive/ Light) but combined with high sedentary proportion. A minority met the recommended MVPA (33\% on weekdays and $20 \%$ on weekends).

Most classes captured similar patterns of physical activity and sedentary behaviour on weekdays and weekends, apart from the Highly active classes which contained children who are more active and less sedentary at the weekend than during the week. The two Active classes were similar in terms of MVPA but differed in time spent in sedentary and light activity, and likewise for the two Inactive classes. In the Highly active and Active classes, the majority met the recommendation of $60+$ mins of MVPA, with more achieving the guidelines at weekends than on weekdays. Only a minority of those in the Inactive classes achieved the recommended MVPA levels, with this proportion higher on weekdays than weekends.

\section{Associations with class membership}

The Highly active classes were predominantly boys (88\%), while the Inactive/sed class comprised $60 \%$ girls (Table 1 ). There were similar proportions of girls and boys in the other classes. The Inactive/light class had a higher level of deprivation (IMD score $=18.9$ 95\% CI: 15.7, 22.2) than other classes. BMI z-score at ages 6 or 9 and average hours of screen viewing were similar across the classes (Table 1).

\section{Age 9 cross-sectional latent profiles}

The BIC for the data (Additional file 1: Table S2) indicated either a 6 or 7 -class model, with the LMR test giving support to a 6-class model. The 7-class model has a slightly lower BIC but produced one very small class (1\%) of highly sedentary and inactive children, similar to that observed for the age 6 data, and we encountered problems with model identifiability due to the small numbers. As the remaining six classes had very similar profiles to the 6-class model, we chose this model, consistent with our approach for the age 6 data.

When labelling the Age 9 classes, we used some of the Age 6 labels to reflect similar profiles. However, these classes are not directly equivalent as nearly all classes are more sedentary at age 9 than at age 6 . Instead we have defined 'similar' in terms of patterns of high and low activity and sedentary proportions, when compared to the overall year average levels. This means, for example, that the Inactive/sed class is always defined as 
Table 1 Age 6: Model-based estimates of covariate means and test for differences across classes

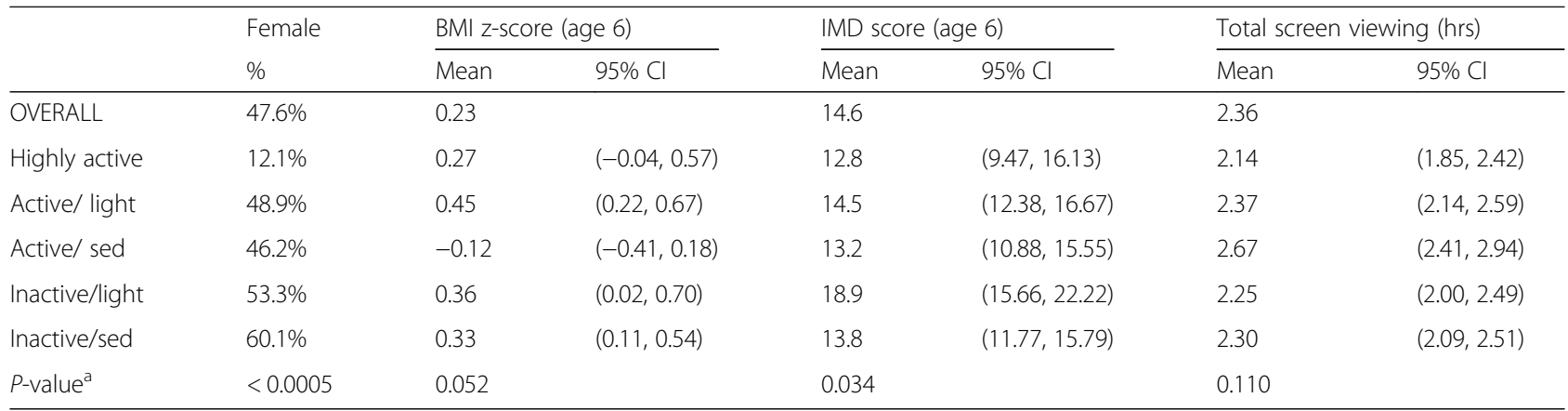

abld test for differences in means across latent classes

being less active and more sedentary than average, however the actual proportion of time spent in sedentary behaviour in this class is higher at age 9 than at age 6 while MVPA remains similar. We have highlighted differences in interpretation of classes below, and the reader is encouraged to keep these differences in mind when making comparisons. The class profiles are summarised in Additional file 1: Table S3 and Fig. 2, and were given the following labels:

- Highly active (7\%): Very similar to the Age 6 Highly active class. High and very high levels of MVPA, especially at weekends, combined with low sedentary time. Nearly all meet the recommended MVPA (90\% on weekdays and $100 \%$ on weekends)

- Active/light (6\%): Similar to the Age 6 Active/light class, but with slightly lower sedentary proportions. Higher MVPA than average and average to low sedentary - more so at weekends. A large proportion of non-sedentary time is spent in light activity rather

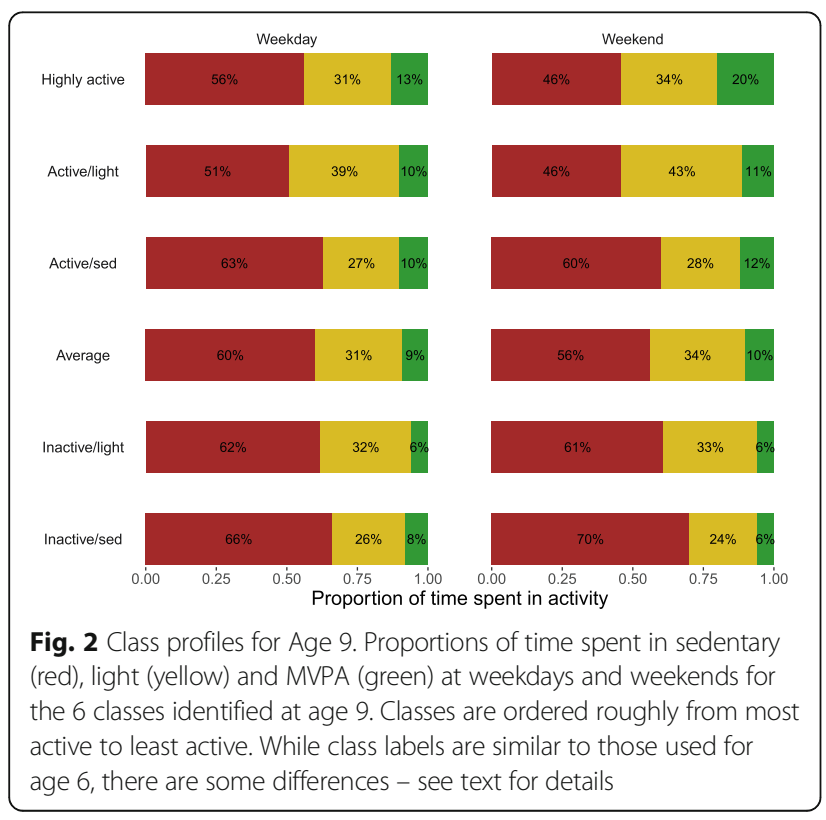

than MVPA. Most meet the recommended MVPA (68\% on weekdays and $78 \%$ on weekends)

- Active/sed (11\%): Very similar to the Active/sed class at age 6. Higher MVPA than average (similar to Active/light), but above average sedentary times more so at weekends. Most meet the recommended MVPA (72\% on weekdays and $91 \%$ on weekends)

- Average (33\%): No corresponding class at age 6. Average levels of sedentary and MVPA; slightly more active and less sedentary at weekends. The majority met the recommended MVPA (59\% on weekdays and $73 \%$ on weekends).

- Inactive/light (22\%): Similar pattern to the Age 6 Inactive/light class, but MVPA is much lower. Very low MVPA combined with average sedentary; a large proportion of non-sedentary time is spent in light activity. A minority meet the recommended MVPA ( $11 \%$ on weekdays and $11 \%$ on weekends)

- Inactive/sed (21\%): Similar to the Age 6 Inactive/ sed class. Low/ very low MVPA (similar to Inactive/ light) but combined with very high sedentary proportions. A minority meet the recommended MVPA (42\% on weekdays and $14 \%$ on weekends)

In contrast to the classes at age 6, we saw slightly stronger differences in weekday/weekend patterns of physical activity and sedentary time within the classes, with all classes except Inactive/sed exhibiting lower sedentary proportions at weekends. The majority of children in the Highly Active, Active/light, Active/sed and Average classes met the recommended levels of MVPA, especially at weekends. Only a minority of those in the two Inactive classes met the recommended MVPA levels, with this proportion higher on weekdays than weekends.

\section{Associations with class membership}

At age 9, all covariates differ between classes. Most participants in the Highly active class were boys (93\%) whereas most in the Inactive/light class were girls (75\%; Table 2). Differences in BMI z-score are driven by the high value for the Active/light class and low value for 
Table 2 Age 9: Model-based estimates of covariate means and test for differences across classes

\begin{tabular}{|c|c|c|c|c|c|c|c|c|c|}
\hline & \multirow{2}{*}{$\begin{array}{l}\% \text { female } \\
\%\end{array}$} & \multicolumn{2}{|c|}{ BMI z-score (age 9) } & \multicolumn{2}{|c|}{ IMD score (age 9) } & \multicolumn{2}{|c|}{ Total screen viewing (hrs) } & \multicolumn{2}{|c|}{ Activity participation } \\
\hline & & Mean & $95 \% \mathrm{Cl}$ & Mean & $95 \% \mathrm{Cl}$ & Mean & $95 \% \mathrm{Cl}$ & Mean & $95 \% \mathrm{Cl}$ \\
\hline OVERALL & $55.2 \%$ & 0.32 & & 15.6 & & 2.89 & & 5.87 & \\
\hline Highly active & $7.1 \%$ & 0.22 & $(-0.02,0.46)$ & 15.3 & $(11.5,19.1)$ & 2.90 & $(2.46,3.34)$ & 7.53 & $(6.91,8.14)$ \\
\hline Active/ light & $44.2 \%$ & 0.81 & $(0.42,1.19)$ & 21.8 & $(15.5,28.1)$ & 2.33 & $(1.78,2.88)$ & 6.25 & $(5.45,7.04)$ \\
\hline Active/ sed & $44.6 \%$ & -0.18 & $(-0.43,0.07)$ & 11.8 & $(9.1,14.5)$ & 2.72 & $(2.35,3.10)$ & 6.14 & $(5.56,6.72)$ \\
\hline Average & $56.2 \%$ & 0.27 & $(0.13,0.42)$ & 15.5 & $(13.4,17.5)$ & 2.70 & $(2.46,2.93)$ & 6.11 & $(5.78,6.44)$ \\
\hline Inactive/light & $75.1 \%$ & 0.56 & $(0.36,0.75)$ & 17.8 & $(15.2,20.5)$ & 3.03 & $(2.71,3.35)$ & 5.38 & $(5.00,5.77)$ \\
\hline Inactive/sed & $55.2 \%$ & 0.36 & $(0.16,0.56)$ & 14.6 & $(12.2,17.0)$ & 3.28 & $(3.00,3.55)$ & 5.13 & $(4.74,5.52)$ \\
\hline$P$-value ${ }^{a}$ & $<0.0005$ & $<0.0005$ & & 0.007 & & 0.007 & & $<0.0005$ & \\
\hline
\end{tabular}

${ }^{a}$ Wald test for differences in means across latent classes

Active/sed. The Active/light class has a higher IMD score, and so contains more individuals with higher deprivation scores than other classes. Average hours of screen viewing differed between classes, and this was mainly due to differences in weekend screen viewing (Additional file 1: Table S4); higher levels of weekend screen viewing were seen in the two Inactive classes, and also in the Highly active class. These average approximately $4 \mathrm{~h}$ of screen-viewing at weekends, compared to $3-3.5 \mathrm{~h}$ for the other classes. There was a strong association between class membership and activity participation, with a difference between the lowest activity in the Inactive/sed class and the highest in the Highly active class of 2.4, which corresponds approximately to an extra four to five sessions of activity per week. When the type of activity was further separated into participation in structured (clubs) and unstructured (playing outdoors) activities (Additional file 1: Table S4) we saw a different pattern in the two Active classes, with Active/ sed higher in structured activity and Active/light higher in unstructured. Those in the Highly active class score high on both.

\section{Changes between profiles at age 6 and age 9 Comparison of age 6 and age 9 profiles}

Figure 3 shows the prevalence of the different classes at age 6 and age 9 , side by side. Fewer children at age 9 are members of the Active classes than at age 6, especially the Active/light, and the new class, Average, is the most commonly occurring class. The proportion of children in the two Inactive classes is approximately the same, but the Inactive/sed class has reduced and Inactive/light increased. MVPA was much lower in the Age 9 Inactive/ light class than either of the Age 6 Inactive classes.

\section{Transition between ages 6 and 9}

We investigated fixing the same latent classes at both time points (measurement invariance model), but this did not fit the data well. This is supported by the cross-sectional findings, which found a different number of latent classes at each age and differences in the class definitions, in particular with higher sedentary proportions at age 9. The results presented here are based on a non-invariance model (classes differ), with latent class profiles fixed to be the same as those found in the cross-sectional analyses.

Figure 4 shows how children moved between Age 6 classes and Age 9 classes using the model-based estimates of the transition probabilities (Additional file 1: Table S5). There was substantial movement between classes between ages 6 and 9, with around 30\% remaining in the similar class. Note that with the exception of the Active/light class, all Age 9 classes were more sedentary than their Age 6 counterparts, and so children who move to a more active class may still become more sedentary. The most common patterns of movement were to classes with either no change or a decrease in MVPA (84\%), and children in active classes at age 6

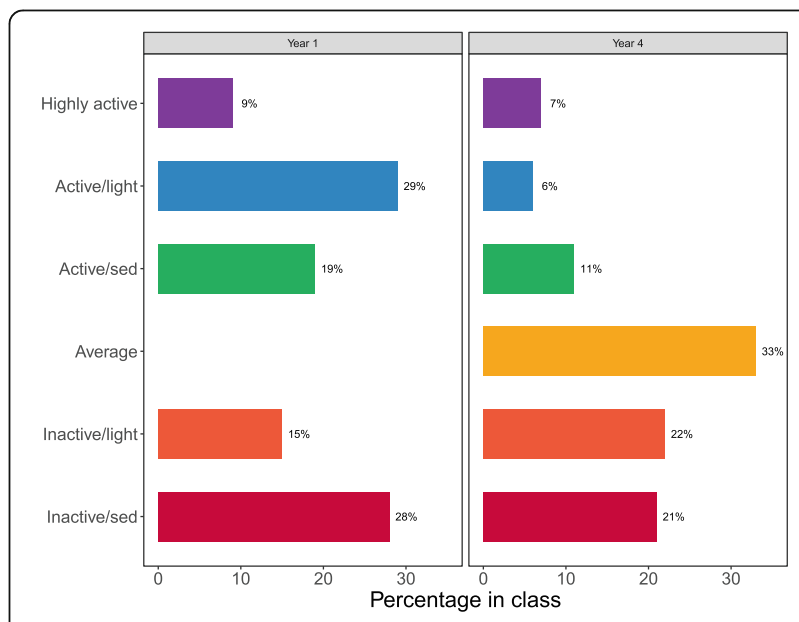

Fig. 3 Comparison of class prevalence between ages 6 (left) and 9 (right). Classes with similar labels show similar patterns at ages 6 and 9 , but there are some differences; see text for details. Remaining classes are seen only in the given year 


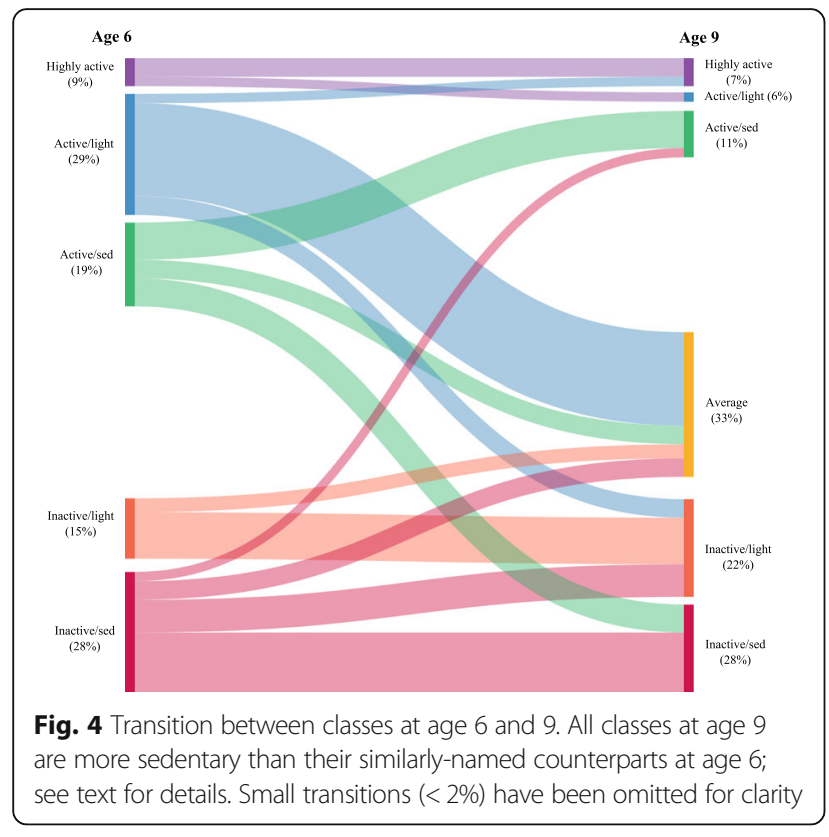

were more likely to remain in active classes at age 9 $(45 \%)$ than children in inactive classes at age 6 were to move to active classes at age $9(12 \%)$. Children who move from Active to Inactive classes tended to keep the same light/sedentary behaviour, while children who moved away from Highly active tended to move to the light rather sedentary classes.

The transition probabilities between classes were associated with all the characteristics that we compared, except deprivation score. Girls had higher probability of moving to classes with lower MVPA than boys (Additional file 1: Table S5). Additional file 1: Figures S1-S3 show how the transition probabilities depend on BMI z-score at age 6, activity participation score and hours of weekend screen viewing. Higher BMI z-score at age 6 was associated with lower probabilities of moving to more active classes and higher probabilities of moving to less active classes. For example, overall, children in the Active/sed class at age 6 had a 43\% probability of staying in the similar Age 9 class and $30 \%$ probability of moving to Inactive/sed. For children with a BMI z-score of 1 (corresponding roughly to a definition of 'Overweight' [31]) these transitions are estimated at $29 \%$ and $38 \%$.

The probability of remaining in the Highly active class between ages 6 and 9 is strongly associated with higher activity participation. A child in the Highly active class at age 6 with a participation score at the average of 6 has a $32 \%$ probability of remaining in the Highly active class, increasing by approximately 8 percentage points for every extra activity session per week (one unit increase in participation score). A higher participation score is also associated with an increase in the probability of moving into the Highly active class at age 9 from other classes. Activity participation was also associated with moving from Inactive classes to Active classes, although the numbers of children transitioning between these two was small. Patterns were similar for associations with structured and unstructured activity (not shown), but with high levels of unstructured activity and low levels of structured activity associated with a move to the Active/light class, and high levels of structured activity associated with movement to the Active/sed class.

Transition probabilities were associated with the average number of hours spent screen viewing at weekends but not on weekdays. The strongest patterns were for the Active/sed and Inactive/sed classes at age 6 where more hours spent screen viewing increased the probability of transitioning to the Inactive/sed class at age 9. These probabilities are 31\% for Active/sed and 47\% for Inactive/sed for an average screen viewing of $3.8 \mathrm{~h}$ at weekends, increasing to $41 \%$ and $58 \%$ for an extra $2 \mathrm{~h}$. In the Highly active class, the opposite pattern was seen, with very high screen-viewing associated with remaining in the Highly active class.

\section{Discussion}

The data presented in this paper have shown that five different classes described the physical activity and sedentary behaviour of pupils at age 6 and six classes at age 9. The profiles range from very active to inactive with differentiation between subgroups in terms of sedentary and light behaviour. Five classes were found to be similar between the 2 years, in terms of general patterns, with the addition of a substantial extra class of 'average' children at age 9 who fell between the more extreme active and inactive classes seen at age 6 . This suggests that the classes are relatively stable, although the proportions in each class change, with the active classes decreasing in prevalence. It is important to note that consistent with a previous study using similar methods applied to physical activity data from several countries [4], all classes were more sedentary at age 9 than at age 6 and that the classes picked up important differences between weekday and weekend physical activity which would not have been apparent by comparing average time in MVPA. Of relevance is that the active classes tended to engage in the recommended $60 \mathrm{~min}$ of MVPA at the weekend, more so than during the week, while inactive classes were unlikely to meet this guideline at either weekends or week days. Recent research has shown that adults who engage in high levels of physical activity at the weekend but are less active on weekdays (i.e., weekend warriors) have reduced risk of cardiovascular disease and all-cause mortality [46], suggesting that weekend activity may be important for health outcomes in adults. Some studies have assessed weekday and weekend differences in children [47], but none have looked at the 
contribution weekend activity makes towards overall MVPA recommendations. Our results suggest that the contribution of weekend activity to an average of 60 min per day of MVPA across the week [48] among children may warrant further investigation.

All Age 9 classes were more sedentary than their Age 6 counterparts. There was substantial movement between classes between age 6 and 9 with less than a third of pupils remaining in the similar class across the two time points. Across this time, the strongest patterns of movement were to profiles with either no change or a decrease in MVPA, and even where children moved to a more active class they typically become more sedentary. This highlights a need to understand the factors that are associated with change in activity profile from age 6 to 9 and how to mitigate movement to less active profiles.

The most robust finding from previous studies is an association between girls and low-activity clusters [22] and this is supported by our findings. Specifically, the Highly active class is predominantly boys at both ages, with the proportion of boys increasing from 88 to $93 \%$. Likewise, at the other end of the scale the Inactive classes have a higher proportion of girls. Gender is also strongly associated with movement between classes with girls more likely to move to less active classes and boys more likely to move to more active classes. Unfortunately, sample sizes were too small to produce separate profiles for boys and girls, especially to look at change over time or associations with covariates. However, the findings do suggest that girls may particularly be at risk of moving to less active profiles.

Participants' body mass-index (z-score), screen-viewing behaviour and the extent to which they engaged in activities were associated with movement between classes. These characteristics were not strongly associated with class membership at age 6 but showed much stronger links with classes at age 9. Movement to more active classes was associated with a lower BMI z-score at age 6 and higher activity participation, with the latter strongly associated with movement into the Highly active class. This indicates that both structured and unstructured activities may contribute to children's overall physical activity levels and maintained engagement in such activities may mitigate some of the age-related decline in activity. Conversely, screen-viewing was associated with becoming more sedentary, rather than becoming less active. For example, in the Highly active class, more screen-viewing was associated with remaining Highly active, suggesting that for this class, screen-viewing occurs in addition to, rather than instead of, being active. This is consistent with a male 'techno-active' group commonly identified in cluster analyses [49]. Collectively, these changes in class membership highlight how screen-viewing, body mass and the types of activities in which children engage can affect their overall physical activity profile. There is strong evidence that sedentary behaviour tracks from childhood to adulthood [50] and that sedentary behaviour is associated with adverse health impacts in adults [7]. The findings therefore suggest that gaining a detailed understanding of the nature of these associations may be important for developing more effective behaviour change programs.

A key finding from this study was that children who are active are more likely to remain active than inactive children to become active. For example, children in the Active/light and Active/sed classes at age 6 have similar MVPA but have distinct patterns of movement. Most of the Active/light class move to the new Average class at age 9; their proportion of non-sedentary time spend in MVPA remains the same but as sedentary time increases, the total MVPA decreases. Meanwhile, children in the Active/sed class are most likely to either remain in Active/sed (more sedentary time, but similar MVPA) or move to Inactive/sed (more sedentary time and less MPVA). In both cases, those who move to Inactive classes tend to keep the same light/sedentary behaviour. This suggests that sedentary patterns reflect underlying behaviour preferences which are independent of how much activity children engage in. This finding suggests that early physical activity behaviours may contribute to physical activity throughout childhood and developing strategies to engage children in physical activity early and then keep them active are likely to be very important. Research that examines this possibility is therefore essential for the advancement of the field.

The differences in movement from the Active/light and Active/sed classes may be due to changes in the amount of structured versus unstructured activity, with parents reporting a decrease in free play between age 6 and 9 [51]. While an activity participation score is not available at age 6 , if we assume the classes have similar activity profiles as at age 9, a key difference between Active/light and Active/sed is that the former class engage in predominantly unstructured activity while the latter in structured activity. A gradual decline in the amount of unstructured activity, when not replaced by structured activities results in a decline in activity levels (i.e., Active/light to the Average class). Conversely, those in the Active/sed class may either continue their club activity and remain in Active/sed, or experience a sudden drop in MVPA when they stop the activity and they transition to the Inactive/sed class. In addition, we note that both Active/sed and Inactive/sed are more likely at age 9 to meet the recommended 60 min of MVPA than their light counterparts, despite having similar MVPA and engaging in more sedentary time. This suggests that unstructured activities may be more variable in the intensity of activity (perhaps more often dipping into light rather than moderate activity), whereas structured 
activity may enable children to more consistently meet MVPA guidelines. This highlights the potential important role of structured activities, such as after-school activity clubs, in promoting and maintaining physical activity in childhood, and if unstructured activity continues to decline may indicate that those in the Average and Active/light classes are at risk of becoming more inactive in the future.

\section{Strengths and limitations}

The major strength of this study is the use of objective physical activity and sedentary behaviour data to examine how physical activity profiles change from Year 1 (age 6) to Year 4 (age 9) of primary school. By applying latent profile analysis to a contextually rich dataset we have provided new information which provides some insights into the change in profiles and the potentially important role of weekend physical activity. The study does however have several limitations that need to be considered. Although the data are from a relatively large cohort, the sample is from a single UK city and some of the transitions are based on a relatively small number of cases. As a result, we are unable to conduct separate analyses for boys and girls, despite the strong associations between gender and latent classes. The class grouping was relatively consistent between the two time points, which suggests a degree of robustness, but the latent class approach is data driven which makes comparisons between studies more difficult. Finally, it is also important to recognise that the move from Year 1 to Year 4 is a key period of change in children's lives when their motor skills develop, they get increased licence to be physically active and there are numerous other social changes, such as going to bed later and increased homework, which can all impact on physical activity and sedentary time [51]. These broader factors may be associated with the profiles detected at the two time points and, aside from the reasons discussed, may help to explain movement in classes across the two timepoints.

\section{Conclusions}

Five profiles were identified at ages 6 and six profiles at age 9, reflecting different patterns of physical activity and sedentary time, and differences between weekdays and weekends. There was substantial movement between profiles between ages 6 and 9, with transitions associated with sex, BMI z-score, screen-viewing and participation in out-of-school activities. Our results highlight the importance of engaging children in physical activity early and the potential important role of structured activities, such as after-school activity clubs, in promoting and maintaining physical activity in childhood. Weekend differences suggest that greater focus on how weekend activity contributes to an average of $60 \mathrm{~min}$ per day of MVPA across the week may be warranted.

\section{Additional file}

Additional file 1: Table S1. Characteristics of sample - observed and missing data. Table S2. Model Fit for models with 2-10 classes. Table S3.

Class membership proportions and percentage time in sedentary, light and MVPA respectively. Table. Age 9: Model-based estimates of additional covariate means and test for differences across classes. Table S5. Estimated transition probabilities: probability a child will move from a profile at age 6 to a profile at age 9. Figure S1. Associations between transition probabilities and BMI z-score at age 6 . How transition probabilities from classes at age 6 (panels) to classes at age 9 (lines) change with BMI z-score at age 6. Figure $\mathbf{S 2}$. Associations between transition probabilities and activity participation. How transition probabilities from classes at age 6 (panels) to classes at age 9 (lines) change with activity participation score. A one unit increase in activity score corresponds to approximately one extra session of activity per week. Figure S3. Associations between transition probabilities and weekend screen-viewing. How transition probabilities from classes at age 6 (panels) to classes at age 9 (lines) change with hours of weekend screen-viewing. (DOCX $397 \mathrm{~kb}$ )

\section{Abbreviations}

BIC: Bayesian Information Criterion; BLRT: Bootstrapped likelihood ratio test; BMI: Body-mass index; IMD: Indices of Multiple Deprivation; LCA: Latent class analysis; LMR: Lo-Mendell-Rubin test; LPA: Latent profile analysis;

MVPA: Moderate-to-vigorous-intensity physical activity

\section{Acknowledgements}

We would like to thank all of the families and schools that have taken part in the B-PROACT1V project. We would also like to thank all current and previous members of the research team who are not authors on this paper.

\section{Funding}

This research was funded by grants from the British Heart Foundation (ref PG/11/51/28986 and SP 14/4/31123). The funder had no involvement in data analysis, data interpretation or writing of the paper. DAL works in a unit that receives funding from the University of Bristol and UK Medical Research Council (MC_UU_1201/5); she is also a UK National Institute of Health and Research Senior Investigator (NF-SI-0166-10196).

\section{Availability of data and materials}

The datasets generated during the current study are not publicly available as the project is ongoing and data are not ready for archiving. We will consider reasonable requests for access to the data once the project is complete in 2019.

\section{Authors' contributions}

RJ, SJS, JLT and DAL were involved in the design of this study and in seeking funding for it. The paper was conceived by RJ and SJS. RS performed all analyses. RJ, RS and SJS wrote the first draft of the paper and RJ coordinated contributions from other authors. All authors made critical comments on drafts of the paper. All authors read and approved the final manuscript.

\section{Ethics approval and consent to participate}

Ethical approval for this study was provided by the School for Policy Studies Research Ethics Committee at the University of Bristol and written parental consent was provided for both parent and child participation.

\section{Consent for publication}

As part of the informed consent process parents provided written consent for publication for both parent and child data.

\section{Competing interests}

The authors declare that they have no competing interests. 


\section{Publisher's Note}

Springer Nature remains neutral with regard to jurisdictional claims in published maps and institutional affiliations.

\section{Author details}

'Centre for Exercise, Nutrition \& Health Sciences, School for Policy Studies, University of Bristol, 8 Priory Road, Bristol BS8 1TZ, UK. ${ }^{2}$ MRC Integrative Epidemiology Unit at the University of Bristol, Oakfield House, Oakfield Grove, Bristol BS8 2BN, UK. ${ }^{3}$ Population Health Sciences, Bristol Medical School, University of Bristol, Canynge Hall, Whiteladies Road, Bristol BS8 2PS, UK. ${ }^{4}$ School of Sport, Exercise and Rehabilitation Sciences, University of Birmingham, Birmingham B15 2TT, UK.

Received: 25 June 2018 Accepted: 9 October 2018

Published online: 23 October 2018

\section{References}

1. Strong WB, Malina RM, Blimkie CJ, Daniels SR, Dishman RK, Gutin B, Hergenroeder AC, Must A, Nixon PA, Pivarnik JM, et al. Evidence based physical activity for school-age youth. J Pediatr. 2005;146(6):732-7.

2. Ekelund U, Luan J, Sherar LB, Esliger DW, Griew P, Cooper A. Moderate to vigorous physical activity and sedentary time and cardiometabolic risk factors in children and adolescents. JAMA. 2012;307(7):704-12.

3. Ekelund U, Steene-Johannessen J, Brown WJ, Fagerland MW, Owen N, Powell KE, Bauman A, Lee IM. Does physical activity attenuate, or even eliminate, the detrimental association of sitting time with mortality? A harmonised meta-analysis of data from more than 1 million men and women. Lancet. 2016;388(10051):1302-10.

4. Cooper AR, Goodman A, Page AS, Sherar LB, Esliger DW, van Sluijs EM, Andersen LB, Anderssen S, Cardon G, Davey R, et al. Objectively measured physical activity and sedentary time in youth: the international children's accelerometry database (ICAD). Int J Behav Nutr Phys Act. 2015;12:113.

5. Jago R, Solomon-Moore E, Macdonald-Wallis C, Sebire SJ, Thompson JL, Lawlor DA. Change in children's physical activity and sedentary time between year 1 and year 4 of primary school in the B-PROACT1V cohort. Int J Behav Nutr Phys Act. 2017;14:33.

6. World Health Organization. Global action plan on physical activity 2018 2030: more active people for a healthier world. Geneva: Licence: CC BY-NCSA 3.0 IGO; 2018.

7. Tremblay MS, Aubert S, Barnes JD, Saunders TJ, Carson V, Latimer-Cheung AE, Chastin SFM, Altenburg TM, Chinapaw MJM, Sedentary behavior research network (SBRN) - Terminology Consensus Project process and outcome. Int J Behav Nutr Phys Act. 2017;14(1):75.

8. Dobbins M, Husson H, DeCorby K, LaRocca RL. School-based physical activity programs for promoting physical activity and fitness in children and adolescents aged 6 to 18. Cochrane Database Syst Rev. 2013;2:CD007651.

9. Biddle SJ, Gorely T, Marshall SJ, Murdey I, Cameron N. Physical activity and sedentary behaviours in youth: issues and controversies. J R Soc Promot Heal. 2004;124(1):29-33.

10. Jago R, Zahra J, Edwards MJ, Kesten JM, Solomon-Moore E, Thompson JL, Sebire SJ. Managing the screen-viewing behaviours of children aged 5-6 years: a qualitative analysis of parental strategies. BMJ Open. 2016;6(3): e010355.

11. Tremblay MS, Carson V, Chaput JP, Connor Gorber S, Dinh T, Duggan M, Faulkner G, Gray CE, Gruber R, Janson K, et al. Canadian 24-hour movement guidelines for children and youth: an integration of physical activity, sedentary behaviour, and sleep. Appl Physiol Nutr Metab. 2016:41(6 Suppl 3):S311-27.

12. Okely AD, Ghersi $D$, Hesketh $K D$, Santos R, Loughran SP, Cliff DP, Shilton $T$, Grant D, Jones RA, Stanley RM, et al. A collaborative approach to adopting/ adapting guidelines - the Australian 24-hour movement guidelines for the early years (birth to 5 years): an integration of physical activity, sedentary behavior, and sleep. BMC Public Health. 2017;17(Suppl 5):869.

13. Hansen BH, Anderssen SA, Andersen LB, Hildebrand M, Kolle E, SteeneJohannessen J, Kriemler S, Page AS, Puder JJ, Reilly JJ, et al. Cross-sectional associations of reallocating time between sedentary and active Behaviours on Cardiometabolic risk factors in young people: an international Children's Accelerometry database (ICAD) analysis. Sports Med. 2018;48(10):2401-12.

14. Stamatakis E, Rogers K, Ding D, Berrigan D, Chau J, Hamer M, Bauman A. Allcause mortality effects of replacing sedentary time with physical activity and sleeping using an isotemporal substitution model: a prospective study of 201,129 mid-aged and older adults. Int J Behav Nutr Phys Act. 2015;12:121.
15. Everitt BS, Landau S, Leese M, Stahl D. Cluster analysis, 5th edition edn. Chichester: John Wiley \& Sons, Ltd; 2011.

16. Collins LM, Lanza ST. Latent class and latent transition analysis : with applications in the social, behavioral, and health sciences. Hoboken, N.J: Wiley; 2010.

17. Masyn K. Latent class analysis and finite mixture modeling. In: Little TD, editor. The Oxford Handbook of Quantitative Methods, vol. 2. Oxford: Oxford University Press; 2013. p. 551-611.

18. Laxer RE, Brownson RC, Dubin JA, Cooke M, Chaurasia A, Leatherdale ST. Clustering of risk-related modifiable behaviours and their association with overweight and obesity among a large sample of youth in the COMPASS study. BMC Public Health. 2017;17(1):102.

19. Cleveland MJ, Lanza ST, Ray AE, Turrisi R, Mallett KA. Transitions in first-year college student drinking behaviors: does pre-college drinking moderate the effects of parent- and peer-based intervention components? Psychol Addict Behav. 2012;26:440-50

20. Leech RM, Timperio A, Livingstone KM, Worsley A, McNaughton SA. Temporal eating patterns: associations with nutrient intakes, diet quality, and measures of adiposity. Am J Clin Nutr. 2017;106(4):1121-30.

21. Lindwall M, Weman-Josefsson K, Sebire SJ, Standage M. Viewing exercise goal content through a person-oriented lens: a self-determination perspective. Psychol Sport Exerc. 2016;27:85-92.

22. Leech RM, McNaughton SA, Timperio A. The clustering of diet, physical activity and sedentary behavior in children and adolescents: a review. Int J Behav Nutr Phys Act. 2014;11:4.

23. Evenson KR, Wen F, Hales D, Herring AH. National youth sedentary behavior and physical activity daily patterns using latent class analysis applied to accelerometry. Int J Behav Nutr Phys Act. 2016;13:55.

24. Liu J, Kim J, Colabianchi N, Ortaglia A, Pate RR. Co-varying patterns of physical activity and sedentary behaviors and their long-term maintenance among adolescents. J Phys Act Health. 2010;7:465-74.

25. Leech RM, McNaughton SA, Timperio A. Clustering of diet, physical activity and sedentary behaviour among Australian children: cross-sectional and longitudinal associations with overweight and obesity. Int J Obes. 2015; 39(7):1079-85.

26. Jago R, Sebire SJ, Wood L, Pool L, Zahra J, Thompson JL, Lawlor DA Associations between objectively assessed child and parental physical activity: a cross-sectional study of families with 5-6 year old children. BMC Public Health. 2014;14:655

27. Jago R, Thompson JL, Sebire SJ, Wood L, Pool L, Zahra J, Lawlor DA. Crosssectional associations between the screen time of parents and young children: differences by parent and child gender and day of the week. Int J Behav Nutr Phys Act. 2014;11:54.

28. Jago R, Bailey R. Ethics and paediatric exercise science: issues and making a submission to a local ethics and research committee. J Sports Sci. 2001;19:527-35.

29. Evenson KR, Catellier DJ, Gill K, Ondrak KS, McMurray RG. Calibration of two objective measures of physical activity for children. J Sports Sci. 2008;26(14): 1557-65.

30. Cole TJ, Bellizzi MC, Flegal KM, Dietz WH. Establishing a standard definition for child overweight and obesity worldwide: international survey. BMJ Open. 2000:320(7244):1240-3.

31. Cole TJ, Freeman JV, Preece MA. Body mass index reference curves for the UK, 1990. Arch Dis Child. 1995;73(1):25-9.

32. Jago R, Macdonald-Wallis C, Solomon-Moore E, Thompson JL, Lawlor DA, Sebire SJ. Associations between participation in organised physical activity in the school or community outside school hours, and neighbourhood play with child physical activity and sedentary time: a cross-sectional analysis. BMJ Open. 2017;7(9):e017588.

33. McLachlan G, Peel D. Finite mixture models. New York: Wiley; 2000.

34. Muthén LK, Muthén BO: Mplus User's Guide. 8th Edn. Los Angeles, CA: Muthén \& Muthén; 1998-2017.

35. Nylund $K$, Asparouhov T, Muthén B. Deciding on the number of classes in latent class analysis and growth mixture modeling: a Monte Carlo simulation study. Structural Equation Modelling. 2007;14(4):535-69.

36. Schwartz G. Estimating the dimension of a model. Ann Stat. 1978;6:461-4.

37. Lo $Y$, Mendell $N$, Rubin D. Testing the number of components in a normal mixture. Biometrika. 2001;88(3):767-78.

38. Yang C. Evaluating latent class analyses in qualitative phenotype identification. Comput Stat Data Anal. 2006;50:1090-104.

39. Celeux G, Saromenho G. An entropy criterion for assessing the number of clusters in a mixture model. J Classif. 1996;13:195-212. 
40. Bakk Z, Vermunt JK. Robustness of stepwise latent class modeling with continuous distal outcomes. Struct Equ Model. 2015;23:20-31.

41. Chung H, Lanza ST, Loken E. Latent transition analysis: inference and estimation. Stat Med. 2008:27:1834-54.

42. Nylund-Gibson K, Grimm R, Quirk M, Furlong M. A latent transition mixture model using the three-step specification. Structural Equation Modelling. 2014;21:1-16

43. Lanza ST, Collins LM. A new SAS procedure for latent transition analysis: transitions in dating and sexual risk behavior. Dev Psychol. 2008;44(2):446-56.

44. Enders CK, Gottschall AC. Multiple imputation strategies for multiple group structural equation models. Struct Equ Model. 2011;18(1):35-54.

45. Enders CK, Bandalos DL. The relative performance of full information maximum likelihood estimation for missing data in structural equation models. Struct Equ Model. 2001;8(3):430-57.

46. O'Donovan G, Lee IM, Hamer M, Stamatakis E. Association of "weekend warrior" and other leisure time physical activity patterns with risks for allcause, cardiovascular disease, and Cancer mortality. JAMA Intern Med. 2017; 177(3):335-42.

47. Jago R, Anderson CB, Baranowski T, Watson K. Adolescent patterns of physical activity differences by gender, day, and time of day. Am J Prev Med. 2005;28(5):447-52.

48. Williamson C, Kelly P, Strain T. Different analysis methods of Scottish and English child physical activity data explain the majority of the difference between the national prevalence estimates. bioRxiv. 2018;408179.

49. Ferrar K, Chang C, Li M, Olds TS. Adolescent time use clusters: a systematic review. J Adolesc Health. 2013;52(3):259-70.

50. Biddle SJ, Pearson N, Ross GM, Braithwaite R. Tracking of sedentary behaviours of young people: a systematic review. Prev Med. 2010;51(5):345-51.

51. Jago R, Solomon-Moore E, Toumpakari Z, Lawlor DA, Thompson JL, Sebire SJ. Parents' perspectives of change in child physical activity \& screenviewing between Y1 (5-6) \& Y4 (8-9) of primary school: implications for behaviour change. BMC Public Health. 2018;18:520.

Ready to submit your research? Choose BMC and benefit from:

- fast, convenient online submission

- thorough peer review by experienced researchers in your field

- rapid publication on acceptance

- support for research data, including large and complex data types

- gold Open Access which fosters wider collaboration and increased citations

- maximum visibility for your research: over $100 \mathrm{M}$ website views per year

At BMC, research is always in progress.

Learn more biomedcentral.com/submissions 